\title{
Un oncogène comme fil d'Ariane vers le gène de la fibrose kystique du pancréas
}

Comme nous l'avons annoncé l'an dernier $\left(\mathrm{m} / \mathrm{s} \mathrm{n}^{\circ} 8\right.$, vol. 1 , p. 440), le gène de la fibrose kystique du pancréas a été localisé sur le chromosome 7, à proximité du gène codant pour l'oncogène met dont le mode d'activation est rapporté dans la nouvelle précédente. $L^{\prime} A D N$ de la lignée $M N N G$ (voir nouvelle précédente) contenant le gène met activé a été transféré dans des fibroblastes de souris "NIH $3 T 3$ " $a$ l'aide d'une technique dite de "transfert de chromosome " [1]. Il s'agit d'introduire dans les cellules à transformer des fragments entiers de chromosome au lieu de l'ADN purifié, comme cela est pratiqué habituellement. L'intérêt de cette méthode est de transférer dans une cellule une large portion de génome comportant un marqueur de sélection connu... et des gènes ou séquences intergéniques inconnus dont on sait seulement sur des arguments génétiques qu'ils sont proches du marqueur de sélection...

Dans le cas rapporté ici, le marqueur de sélection est l'oncogène met activé, qui provoque l'apparition de foyers de prolifération ( $\mathrm{m} / \mathrm{s} \mathrm{n}^{\circ} 7$, vol. 2, p. 405). Ces foyers ont été "sousclonés" pour obtenir des populations cellulaires dérivées d'une seule cellule transformée, puis injectés dans la souris immunodéprimée Nude, ayant pour effet l'apparition de tumeurs.

Une analyse de l'ADN humain intégré dans les cellules tumorales des souris révéla que sa taille variait de 2 à 10 $\times 10^{6}$ kilobases et, dans la majorité des cas, semblait réarrangée par rap- port à l'organisation normale du génome humain.

Dans un cas, cependant, les $5 \times$ $10^{6}$ kilobases intégrées semblaient non réarrangées. Ce fragment de génome humain étant composé d'ADN génétiquement lié au gène met, et donc au locus de la fibrose kystique du pancréas, il pourrait permettre de découvrir des fragments plus proches du locus morbide que met lui-même... et même, avec un peu de "chance", le gène de la fibrose kystique lui-même.

A.K.

1. Scambler PJ, Law HY, Williamson R, Cooper CS. Chromosome-mediated gene transfer of six DNA markers linked to the cystic fibrosis locus on human chromosome seven. Nucl Acids Res 1986 ; 14 : 7159-14.

\section{Les deux récepteurs du glucagon}

\section{L'AMPC ne serait pas le " second messager " principal du glucagon}

S'il est une certitude qui était bien ancrée dans l'esprit des endocrinologues et des physiologistes, c'est que le mode d'action du glucagon se faisait uniquement via l'activation de l'adénylate cyclase et la synthèse d'AMP cyclique. Un article récent de Wakelam et al. [1] vient de montrer que, dans les conditions physiologiques, il n'en était peut-être rien.

Le TH-glucagon est un analogue de l'hormone qui est incapable de stimuler l'adénylate cyclase mais qui, cependant, stimule complètement la glycogénolyse (hydrolyse du glycogène en glucose), la néoglucogènese (synthèse de glucose à partir de métabolites des acides aminés) et la synthèse de l'urée au niveau des hépatocytes. Au cours du traitement par le THglucagon, la concentration cellulaire de l'AMPc ne change pas mais celle des inositol-phosphates $m / s n^{\circ} 1$ ool. 3, janvier 87 augmente. La constante d'activation de la production d'inositolphosphate par le glucagon ou le $\mathrm{TH}$-glucagon est voisine de $0,3 \mathrm{nM}$. alors que celle de la production d'AMPc par le glucagon n'est que de 6,3 nM.

A faible dose, le glucagon provoque une " désensibilisation " vis-àvis de son effet ultérieur sur l'activation de l'adénylate cyclase, alors qu'à forte dose il provoque une désensibilisation vis-à-vis de la production ultérieure d'inositolphosphate (figure 1). Ainsi existerait-il deux types de récepteurs du glucagon (figure 1).

La stimulation de l'un, que nous appellerons récepteur de type 1 par homologie avec les récepteurs de la vasopressine qui sont également de deux types, entraîne l'activation de la phospholipase C, l'hydrolyse du phosphatidylinositol 4,5 diphosphate en inositol 3 phosphate $\left(\mathrm{IP}_{3}\right)$ et en diacylglycérol (DG).

L'IP ${ }_{3}$ entraîne une libération de calcium des citernes de réticulum endoplasmique, le calcium pouvant être le " troisième messager " responsable des effets métaboliques lors de la stimulation à faible dose de glucagon... comme cela est le cas dans les conditions physiologiques. Le DG stimule la protéine kinase $\mathrm{C}$ qui pourrait, par phosphorylation, inactiver la protéine Gs responsable de l'activation de l'adénylate cyclase. Le récepteur de type 2 serait impliqué à des concentrations supérieures de glucagon, conduisant à la stimulation de l'adénylate cyclase via une protéine Gs, comme indiqué dans la nouvelle de $\mathrm{m} / \mathrm{s} n^{\circ} 10$, vol. 2, p. 583. L'activation de Gs pourrait inhiber la phospholipase C [2].

Si les faits rapportés par l'article de Wakelam et al. [1] semblent 


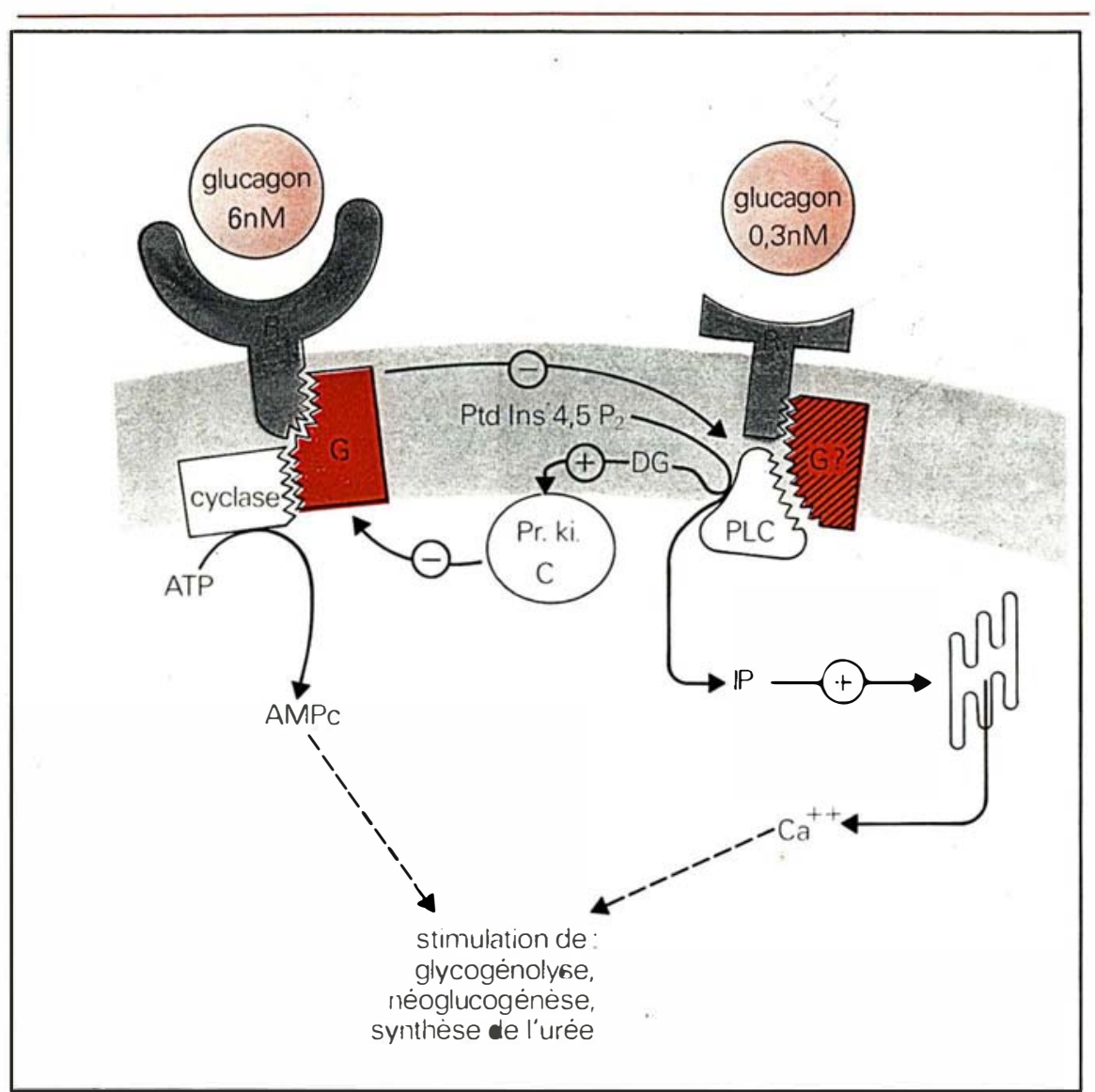

Figure 1. Les deux types de récepteur du glucagon et lours rôles. Cyclase $=$ adénylate cyclase ; Pr. ki. $C=$ protéine kinase PLC = phospholipase $C$; Ptd Ins 4,5 $p_{2}=$ phosphatidyl-Inositol 4,5 diphosphate; $D G=$ diacylglycérol ; $\mathbb{P}_{3}=$ inositol triphosphate; $R_{1}$ et $R_{2}=$ récepteurs du glucagon de type 1 et $2 ; G s=$ protéine $G s ; G ?=$ protéine $G$ hypothétique.

A faible concentration en glucagon, la stimulation de $R_{1}$ provoque l'activation de la PLC qui hydrolyse le Ptd ins $4,5 P_{2}$ en DG et $\mathbb{P}_{3}$. $L^{\prime} I P_{3}$ provoque la sortie de calcium ionisé $\left(\mathrm{Ca}^{++}\right)$des citernes de réticulum endoplasmique. Le $\mathrm{Ca}^{++}$serait ici le "troisième messager " de l'action hormonale. Le DG stimule la Pr. ki. C qui pourrait phosphoryler et inactiver Gs, provoquant la "désensibilisation" du système aboutissant à l'activation de l'adénylate cyclase.

A plus forte concentration de glucagon, les récepteurs $R_{2}$ sont stimulés, activant, via la protéine Gs, l'adénylate cyclase et donc la synthèse d'AMPc, second messager de l'action hormonale. Gs activé pourrait désensibiliser la PLC vis-à-vis de la stimulation des récepteurs $R_{1}$.

indiscutables, de nombreux travaux ultérieurs seront nécessaires pour comprendre la signification de l'existence de ces deux systèmes de stimulation hormonale aboutissant apparemment aux mêmes conséquences métaboliques, les conditions de mise en jeu de l'un ou de l'autre, et leurs interactions. De même, il sera passionnant de déterminer si une telle dualité de récepteurs et de est également vraie pour les nombreuses autres hormones considérées comme agissant exclusivement via l'activation de l'adénylate cyclase.

A.K.

1. Wakelam MJO, Murphy GJ, Hruby VJ, Houslay MD. Activation of two signaltransduction systems in hepatocytes by glucagon. Nature 1986 ; 323 : 68-70.

2. Petersen OH, Bear C. Two glucagon transducing systems. Nature $1986 ; 323: 18$.

\section{口ם BRÈVES}

Une transplantation de moelle osseuse chez des chiens adultes atteints d'une maladie de surcharge, la fucosidose, a montré qu'une activité enzymatique notable se retrouvait dans les cellules nerveuses des receveurs. Ces résultats suggèrent qu'un traitement précoce par greffe de moelle chez des malades porteurs de maladies de surcharge affectant le système nerveux central pourrait peut-être se montrer efficace dans certains cas.

[Taylor RM et al. Lancet 1986 ; ii : 772-4.]

Le facteur natriurétique cardiaque est synthétisé non seulement dans les oreillettes mais aussi dans les ventricules chez le rat. C'est ce que viennent de démontrer A.-L. Lattion et al, en utilisant une sonde complémentaire de l'ARN messager codant pour la portion C-terminale du facteur natriurétique. Cependant, l'ARN messager est environ cinquante fois moins abondant dans les ventricules que dans les oreillettes. Dans quatre modèles expérimentaux différents, où augmente le volume plasmatique, I'ARN messí ger s'élève dans les oreillettes et les ventricules, principalement le ventricule gauche.

[Lattion AL et al. Am J Physiol 1986, sous presse.]

La culture de cellules musculaires humaines vient de faire un progrès décisif. Jusqu'à présent, bien que formant des myotubes, ces cellules ne contenaient que des isoenzymes de type fotal. En les faisant innerver in vitro par des explants de moelle épinière de rat, on obtient des fibres qui, non seulement, se contractent, mais possèdent aussi les protéines et isoenzymes spécifiques du muscle adulte. L'étude in vitro des maladies musculaires devrait donc devenir enfin possible.

[Martinuzzi A et al. J Cell Biol 1986 ; 103 : 1423-9.] 